\title{
Existence and nonexistence of global solutions for logarithmic hyperbolic equation
}

\author{
Yaojun $\mathrm{YE}^{1}$ \\ ${ }^{1}$ Zhejiang University of Science and Technology
}

February 23, 2021

\begin{abstract}
In this paper, we study the initial-boundary value problem of a class of degenerate quasilinear hyperbolic equation with logarithmic nonlinearity. By applying Galerkin method and the logarithmic Sobolev inequality, we prove the existence of global weak solutions for this problem. Meanwhile,the global nonexistence of solutions is verified by means of the concavity analysis when the initial energy is positive and appropriately bounded.
\end{abstract}

\section{Hosted file}

20210201.pdf available at https://authorea.com/users/397582/articles/510422-existence-andnonexistence-of-global-solutions-for-logarithmic-hyperbolic-equation 